\title{
Nuevos registros del gato colocolo, Leopardus colocolo colocolo (Carnivora: Felidae), en el desierto de Atacama, Región de Antofagasta, Norte Grande de Chile
}

\author{
New records of the pampas cat, Leopardus colocolo colocolo (Carnivora: Felidae), in \\ the Atacama Desert, Antofagasta Region, Norte Grande of Chile
}

\author{
Jaime R. Rau ${ }^{1,2, *}$, Carlos Zuleta ${ }^{3}$, Alberto Gantz ${ }^{1}$, J. Agustín Iriarte ${ }^{4}$ \\ ${ }^{1}$ Laboratorio de Ecología, Departamento de Ciencias Biológicas y Biodiversidad \& \\ ${ }^{2}$ Programa IBAM, Universidad de Los Lagos, Casilla 933, Osorno, Chile. \\ ${ }^{3}$ Laboratorio de Ecología de Vertebrados, Departamento de Biología, Facultad de Ciencias, Universidad de La Serena, Casilla \\ 599, La Serena, Chile. \\ ${ }^{4}$ Flora \& Fauna Chile, Magnere 1540, Oficina 506 (esq. Santa Beatriz), Providencia, Santiago.
}

*jrau@ulagos.cl

\begin{abstract}
RESUMEN
Registramos en Quebrada Jerez, Toconao (23 $11^{\prime}$ S y $67^{\circ} 59^{\prime} \mathrm{O}, 2475$ m.s.n.m.), desierto de Atacama (Norte Grande de Chile), la captura de un gato colocolo hembra. Esta captura más nuevos registros con trampas cámaras confirman para la subespecie Leopardus colocolo colocolo su presencia en la Región de Antofagasta. Presentamos datos biométricos y craneométricos y confirmamos por una radiografía de su dentición el estatus juvenil de este felino.
\end{abstract}

Palabras clave: Desierto de Atacama, radiografía de los caninos, felinos, distribución

\begin{abstract}
We report the capture of a female pampas cat in Quebrada Jerez, Toconao ( $23^{\circ} 11^{\prime} \mathrm{S}$ y $67^{\circ} 59^{\prime} \mathrm{O}, 2475$ m.s.n.m.), Atacama desert (Norte Grande of Chile). This capture and more records with camera traps confirm the presence of the subspecies Leopardus colocolo colocolo in the Antofagasta Region. We provide biometric and craniometric data on the specimen and confirm by a canine radiography the juvenile status of this felid.
\end{abstract}

KEYwords: Atacama Desert, distribution, canine radiography, felids

El gato colocolo, Leopardus colocolo (Molina 1782), presenta once subespecies distribuidas en tres especies: Leopardus colocolo, L. pajeros y L. braccatus (Iriarte \& Jaksic 2012). De acuerdo con Iriarte et al. (2011) e Iriarte \& Jaksic (2012) la subespecie chilena sería Leopardus colocolo colocolo la que en la Región de Antofagasta se encuentra en el altiplano, entre los 3000 y 5000 m.s.n.m. Según datos morfológicos García-Perea (1994) postula que esa forma sería Lynchailurus colocolo wolffsohni. Sobre la base de evidencias genéticas recientes su clasificación no es actualmente aceptada (Johnson et al. 1999).

Actualmente se propone cambiar el estado de conservación nacional del gato colocolo a la categoría Cercana a la Amenaza (NT, Iriarte \& Jaksic 2012).
La existencia en Chile para esta subespecie se presumía sólo para la provincia de Tarapacá, limítrofe con la provincia de Antofagasta (Mann 1945, Spotorno et al. 1998).

En octubre de 2007 se capturó una hembra juvenil de gato colocolo, en la localidad de Quebrada Jerez al interior del desierto de Atacama. Este animal se encontraba en buena condición física. El sitio de captura es un oasis que se encuentra ubicado en Toconao (23 $11^{\prime} \mathrm{S}$ y $67^{\circ} 59^{\prime} \mathrm{O}, 2475$ m.s.n.m.), distante 38 km. al sur de San Pedro de Atacama, desierto de Atacama, Región de Antofagasta, Norte Grande de Chile. Este desierto se caracteriza por ser uno de los más áridos y antiguos del mundo (e.g., Clarke 2006).

Aunque la actividad de esta subespecie es nocturna 
(Napolitano et al. 2008) se pudo observar de día y por más de media hora, cercana a una de las riberas del estero Toconao. Durante ese tiempo se la vio alimentarse de 3 zorzales cuyanos, Turdus chihuanco (Lafresnaye \& D'Orbigny 1837) (Passeriformes: Muscicapidae), y aunque exhibió conductas de defensa típicas (Iriarte \& Jaksic 2012), se pudo obtener fotografías y su captura (Fig. 1).

Además de esta captura, los nuevos registros con trampas cámaras (e.g., Lucherini et al. 2009) (Fig. 2) son los siguientes: Quebrada de Turipite (22 $39^{\circ} \mathrm{S}$ y $68^{\circ} 02^{\prime} \mathrm{O}$, 3973 m.s.n.m.), Quebrada de Puritama (22 $43^{\circ} \mathrm{S}$ y $60^{\circ} 03^{\prime}$ O, 3520 m.s.n.m.), Quebrada alta de Puritama $\left(22^{\circ} 40^{\prime} \mathrm{S}\right.$ y $68^{\circ} 00^{\prime}$ O, 3804 m.s.n.m.) y Quebrada de Guanacos $\left(24^{\circ}\right.$ 35 S y 69 03’ O, 2996 m.s.n.m.). También contamos con observaciones confirmadas y recientes para las localidades de Machuca (22 ${ }^{\circ}$ 58' S y $68^{\circ}$ 06' O, 4000 m.s.n.m., fotografía), Aguas Calientes (25 $00^{\prime} \mathrm{S}$ y $68^{\circ} 63^{\prime} \mathrm{O}, 4020$ m.s.n.m., fotografías), Quebrada de Puritama (22 $43^{\prime} \mathrm{S}$ y $60^{\circ} 03^{\prime}$ O, 3520 m.s.n.m. video; Nicolás Melo, com. pers.), Tulor $\left(22^{\circ} 98^{\prime} \mathrm{S}\right.$ y $68^{\circ} 23^{\prime} \mathrm{O}, 2480$ m.s.n.m., fotografía; Alfonso Tapia, com. pers.) y Monturaqui (24 $35^{\prime} \mathrm{S}$ y $68^{\circ}$ 45' O, 3450 m.s.n.m., animal muerto). La distribución altitudinal de Leopardus colocolo colocolo en la Región de Antofagasta se extendería entonces entre los 2475 y 3973 m.s.n.m. Hasta ahora solamente existían dos ejemplares registrados para la provincia de Tarapacá en Chile (GarcíaPerea 1994): un animal adulto de sexo indeterminado

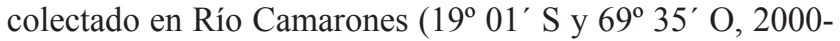

4000 m.s.n.m.) y un macho juvenil colectado en Putre $\left(18^{\circ}\right.$ $12^{\prime} \mathrm{S}$ y $69^{\circ} 35^{\prime} \mathrm{O}, 4120$ m.s.n.m.).

Una vez capturado el animal se le mantuvo en una jaula con agua y se le alimentó ad libitum para ser enviado al Laboratorio de Citogenética Evolutiva de la Universidad de Chile, Santiago pero murió durante el transporte aéreo. Se encuentra depositado allí con el número de colección LCM-1764. Los datos biométricos obtenidos fueron los siguientes: peso $=971 \mathrm{~g}$, largo total $=600 \mathrm{~mm}$, longitud de la cola $=205$, longitud de la pata posterior $=92$, longitud de la oreja $=45$, dentición $(\mathrm{ICPM})=0,1,1,2-3,1,2,0$, edad $=2-4$ meses. Los datos craneométricos fueron: longitud (long.) total craneal $=79,96$, long. condilobasal $=73,61$, long. basal $=65,12$, ancho rostral máximo $=20,12$, long. premolar (PM) en cíngulo-derecho (D) =9,62, long. PM en cínguloizquierdo $(\mathrm{I})=9,97$, long. palatal $=29,73$, ancho mastoideo $=37,27$, ancho cigomático máximo $=53,32$, ancho cráneo máximo $=42,7$, long. cresta sagital $=29,22$, long. máxima nasales-D $=21,33$, long. máxima nasales- $\mathrm{I}=20,43$, ancho interorbitario mínimo $=13,89$, long. mandibular $=50,7$, long. serie dentaria alveolar inferior $=18,49$, long. serie dentaria alveolar superior-I $=20,46$, long. serie dentaria alveolar superior-D $=19,77$, long. máxima $\mathrm{PM}$ en corona- $\mathrm{I}=9,86$, long. máxima PM en corona-D $=9,61$, ancho postorbitario mínimo $=28,4$. Sólo existen datos craneométricos para un adulto de sexo indeterminado (García-Perea 1994), no comparables con los de nuestro ejemplar.

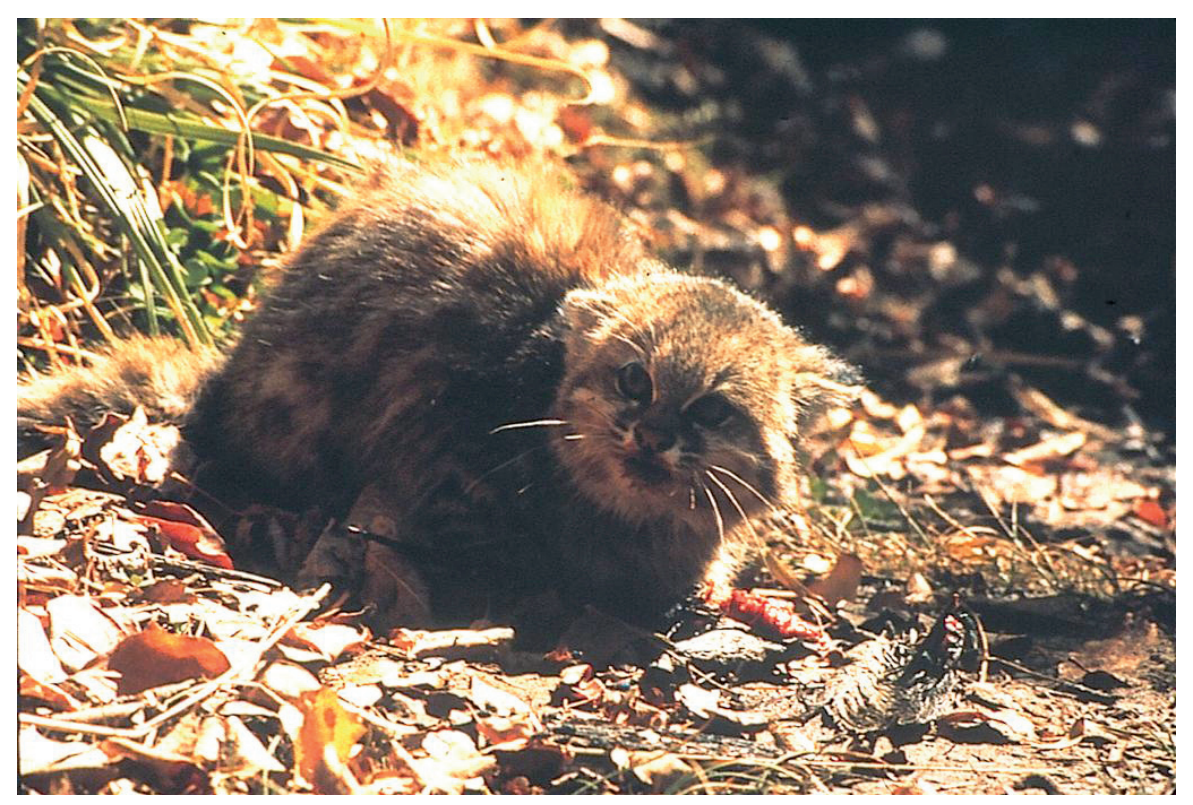

Figura 1. Fotografía de una hembra juvenil de Leopardus colocolo colocolo alimentándose de zorzales cuyanos (Turdus chihuanco) en quebrada Jerez, desierto de Atacama, Región de Antofagasta, Norte Grande de Chile (fotografía tomada por A. Gantz).

FIGURE 1. Photograph of a Leopardus colocolo colocole juvenile female feeding on Chiguanco thrushes (Turdus chihuanco) in Jerez ravine, Atacama desert, Antofagasta region, Norte Grande of Chile (photograph by A. Gantz). 
Para confirmar su estatus juvenil se realizó una radiografía del cráneo para corroborar si la cavidad de la pulpa de sus caninos estaba llena o vacía de dentina y el canal de la raíz abierto (Figs. 3 y 4). Ambos criterios han sido utilizados para diferenciar cánidos juveniles de adultos (Tumlison \& McDaniel 1984). La técnica radiográfica también ha demostrado ser de utilidad para felinos (Berg 1979, Zapata et al. 1997). A través de ella pudimos confirmar que el animal correspondió a un juvenil (Fig. 4), dado que la cavidad de la pulpa estaba abierta y no se apreció la capa de dentina en su dentición.

Recientemente, utilizando trampas cámaras se amplió aún más el área de distribución del gato colocolo a la región costera de la provincia del Huasco, Atacama,

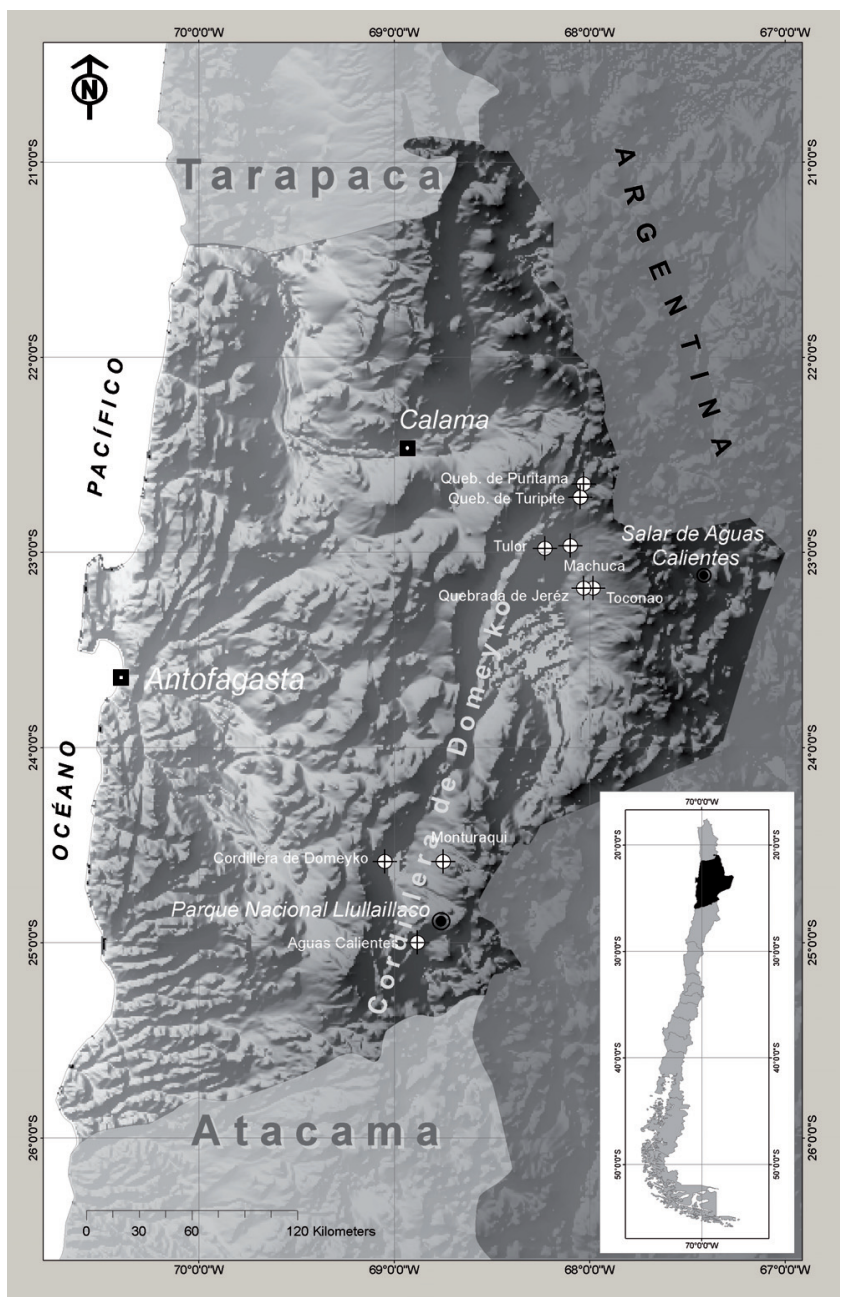

Figura 2. Nuevos registros para la distribución de Leopardus colocolo colocolo en la Región de Antofagasta, Norte Grande de Chile (tomado de Iriarte et al. 2011).

FIGURE 2. New records for the distribution of Leopardus colocolo colocolo in the Antofagasta Region, Norte Grande of Chile (based on Iriarte et al. 2011). específicamente en el Parque Nacional Llanos del Challe (Cepeda-Mercado et al. 2014, Espinoza et al. 2014). También se ha aumentado considerablemente el número de registros y se ha ampliado el área de distribución del gato andino, Leopardus jacobita (Cornalia, 1865), hasta la cordillera de Santiago (A. Iriarte, datos no publicados), especie simpátrica con el gato colocolo. Estas ampliaciones en la distribucion de ambas especies de felinos pueden ser el

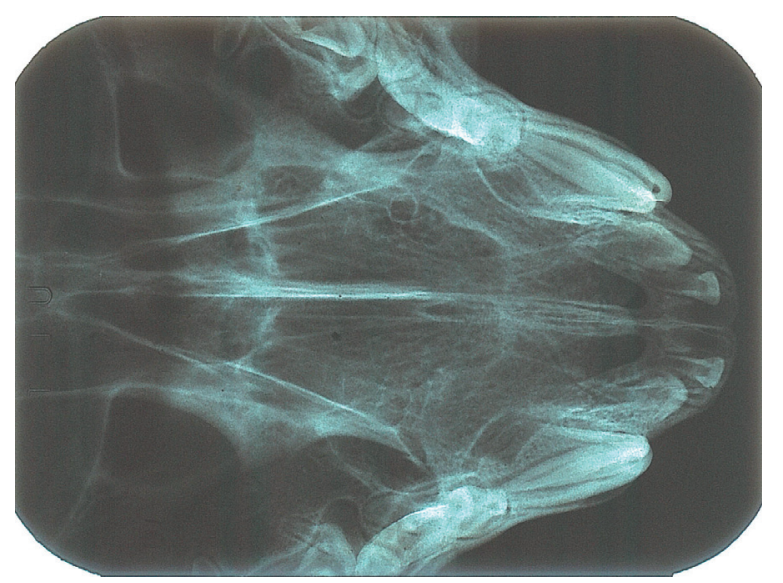

Figura 3. Radiografía (nótense los caninos de leche) de la parte anterior del cráneo de Leopardus colocolo colocolo de quebrada Jerez, desierto de Atacama, Región de Antofagasta, Norte Grande de Chile.

FIGURE 3. Radiography (notice milky canines) of the front part of Leopardus colocolo colocolo skull of Jerez ravine, Atacama desert, Antofagasta Region, Norte Grande of Chile.

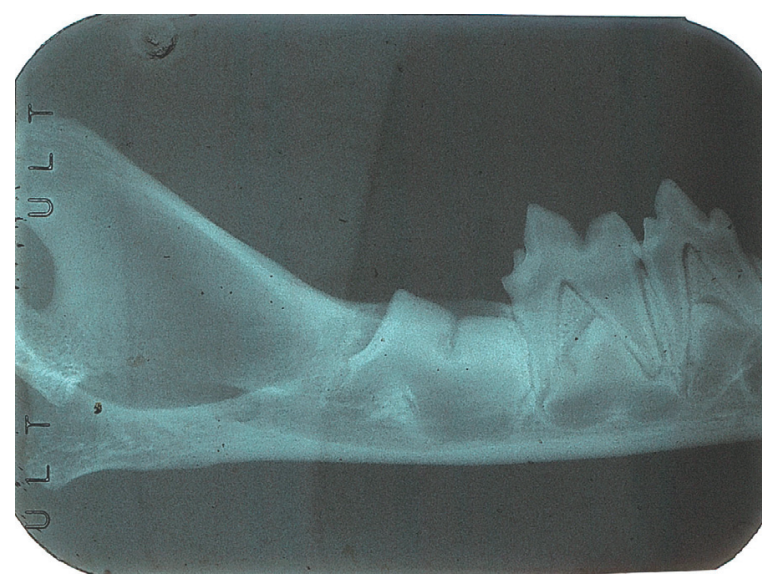

FIgURA 4. Radiografía de la mandíbula inferior izquierda de Leopardus colocolo colocolo de quebrada Jerez, desierto de Atacama, Región de Antofagasta, Norte Grande de Chile. Apréciense en los molares inferiores la cavidad de la pulpa abierta y la ausencia de la capa de dentina en su dentición.

Figure 4. Radiography of left inferior mandible of Leopardus colocolo colocolo of Jerez ravine, Atacama desert, Antofagasta Region, Norte Grande of Chile. Notice in the lower molars the open pulp cavity and the absent oh the dentine layer in this teeth. 
resultado del aumento del esfuerzo de muestreo dado por la implementación reciente en el país de muestreos intensivos con trampas cámaras. Otra explicación puede deberse al calentamiento global. En efecto, Marino et al. (2011) han encontrado que modelos bioclimáticos para gatos andinos fueron altamente predictivos con sólo 3 a 4 variables (tres medidas de la temperatura y la aridez). Más investigación es necesaria para corroborar o refutar ambas hipótesis.

\section{AGRADECIMIENTOS}

Agradecemos a Pablo Campos Botarro por las radiografías y al proyecto Fondecyt No 1980017. Al Servicio Agrícola y Ganadero (SAG) por el permiso de captura científica y a Mateo Rau C. y Soraya Sade por su ayuda en la edición del texto.

\section{BIBLIOGRAFIA}

BERG, W. E. 1979. Ecology of bobcats in northern Minnesota. In: Proceedings of the Bobcat Research Conference (Eds. Blum, L.G. \& Escherich, P.C. ), pp. 55-61. National Wildlife Federation Scientific and Technical Series 6, Washington, D.C., EE.UU.

Cepeda-Mercado A.A., Troncoso, I., Villegas, A., Paredes, L., Araya, S., Ortuya, R., Louit, C., Melendez, M. \& Gonzalez-Maya, J.F. 2014. Felinos del Parque Nacional Llanos del Challe: nuevos retos para la conservacion de la biodiversidad en la Región de Atacama. Biodiversidata 2:104-106.

Clarke, J. D. A. 2006. Antiquity of aridity in the Chilean Atacama desert. Geomorphology 73:101-114.

Espinoza, M., Cepeda-Mercado, A.A., Louit, C., Melendez, M. \& GonzÁlez-Maya, J.F. 2014. Pampas cat Leopardus colocolo in the Atacama desert: first records from Llanos del Challe, National Park, Chile. Boletín del Museo Nacional de Historia Natural, Chile. 63:111-118.

Garcia-Perea, R. 1994. The Pampas cat group (genus Lynchailurus Severtzov, 1858) (Carnivora: Felidae), a systematic and biogeographic review. American Museum Novitates 3096:1-36.
Johnson, W.E, Slattery, J.P., Eizirik, E., Kim, J.H., Raymond, M.M., Bonacic, C., Cambre, R., Crashaw, P., Nunes, A., Seuanez, H.N., Moreira, M.A.M., Seymour, K.L., Simon, F., Swanson, W.\& O' Brien, S.J. 1999. Disparate phylogeographic patterns of molecular genetic variation in four closely related South American small cat species. Molecular Ecology 8:S79-S94.

Iriarte, A. \& JAKSIC, F. 2012. Los carnívoros de Chile. Ediciones Flora \& Fauna Chile y CASEB P. U. Católica de Chile. Santiago, Chile. 260 pp.

Iriarte, A., Lagos, N. \& Villalobos, R. 2011. Los mamíferos de la Región de Antofagasta. Ediciones Flora y Fauna. Santiago, Chile. 332 pp.

Lucherini, M., Repucci, J.I., Walker, R.S., Villalba, M.L., Wurstten, A., Gallardo, G., Iriarte, A., Villalobos, R. \& Perovic, P. 2009. Activity pattern segregation of carnivores in the high Andes. Journal of Mammalogy 90:1404-1409.

Mann, G. 1945. Mamíferos de Tarapacá. Observaciones realizadas durante una expedición al Alto Norte de Chile. Biológica 2:23-138.

Marino, J., Benett, M., Cossios, D., Iriarte, A., Lucherini, M., Pliscoff, P., Villalba, C., Sillero-Zubiri, L. \& Walker, S. 2011. Bioclimatic constrains to Andean Cat distribution: a modelling application for rare species. Diversity and Distributions 17:311-322.

Napolitano, C., Bennett, M., Johnson, W.E., O' Brien, S.J., Marquet, P.A., Barria, I., Poulin, E. \& Iriarte, A.2009. Ecological and biogeographical inferences on two sympatric and enigmatic Andean cat species using genetic identification of faecal samples. Molecular Ecology 17:678-690.

Spotorno, A.E., Zuleta, C., Gantz, A., Saiz, F., Rau, J., Rosenmann, M., Cortes, A., Ruiz, G., Yates, L., Couve, E. \& Marin, J.C. 1998. Sistemática y adaptación de mamíferos, aves e insectos fitófagos de la Región de Antofagasta, Chile. Revista Chilena de Historia Natural 71:501-526.

Tumlison, R. \& McDaniel, V.R. 1984. Gray fox age classification by canine tooth pulp cavity radiographs. Journal of Wildlife Management 48:228-230.

Zapata, S.C., Garcia-Perea, R., Beltran, J.F., Ferrera, P. \& Delibes, M. 1997. Age determination of Iberian lynx (Lynx pardinus) using canine radiograph and cementum annuli enumeration. Zeitschrift für Säugetierkunde 62:119-123.

Recibido: 07.03 .13

Aceptado: 13.03 .15 